\title{
An exploratory intervention study suggests clinical benefits of training in chronic stroke to be paralleled by changes in brain activity using repeated $\mathrm{fMRI}$
}

\author{
This article was published in the following Dove Press journal: \\ Clinical Interventions in Aging \\ 28 January 2016 \\ Number of times this article has been viewed
}

\author{
Barbara Landsmann ${ }^{1,2}$ \\ Daniela Pinter ${ }^{2}$ \\ Eva Pirker ${ }^{1,2}$ \\ Gerald Pichler ${ }^{3}$ \\ Walter Schippinger ${ }^{3}$ \\ Elisabeth MWeiss' \\ Gabriel Mathie ${ }^{2}$ \\ Thomas Gattringer ${ }^{2}$ \\ Franz Fazekas ${ }^{2}$ \\ Christian Enzinger ${ }^{2,4}$ \\ 'Institute of Psychology, University of \\ Graz, Graz, Austria; ${ }^{2}$ Department \\ of Neurology, Medical University \\ of Graz, Graz, Austria; ${ }^{3}$ Albert \\ Schweitzer Clinic Graz, Graz, \\ Austria; ${ }^{4}$ Division of Neuroradiology, \\ Department of Radiology, Medical \\ University of Graz, Graz, Austria
}

\begin{abstract}
Purpose: Previous studies demonstrated changes in sensorimotor network activation over time after stroke that have been interpreted as partly compensatory. Locomotor and balance trainings may improve both mobility and cognition even in chronic stroke and thereby impact on cerebral activation patterns. We here aimed at testing these assumptions in an exploratory study to inform subsequent larger intervention studies.
\end{abstract}

Patients and methods: Eight patients ( $73.3 \pm 4.4$ years) with a chronic lacunar stroke (mean interval 3.7 years after the acute event with a range from 2 to 4 years) and residual leg paresis leading to gait disturbance received a guided 5-week training focusing on mobility, endurance, and coordination. Before and afterward, they underwent clinical, neuropsychological, and gait assessments and brain MRI at $3 \mathrm{~T}$ including a functional ankle movement paradigm. Sixteen healthy controls (HCs; 68.8 \pm 5.4 years) followed the same protocol without intervention.

Results: After training, patients had improved in mobility, memory, and delayed recall of memory. While cerebral activations in HC remained completely unaltered, patients showed increased activations in the right precentral gyrus, the right and left superior frontal gyri, and the right frontal lobe, with bipedal ankle movements after training.

Conclusion: In this exploratory study of chronic stroke, we found not only significant effects of physical training on mobility but also distinct aspects of cognition already with a small number of highly selected patients. These improvements were paralleled by alterations in cerebral activity possibly reflecting neuronal plasticity. Larger studies including randomization are needed.

Keywords: mobility, cognition, lacunar stroke, training, rehabilitation, neuronal plasticity

\section{Introduction}

There are reports that physical training may have positive effects even with more advanced age and in the chronic phase of a stroke. ${ }^{1}$ Such effects have been shown not only concerning mobility but also for cognition, and it has been suggested that training may partially normalize brain activation in motor networks after stroke. ${ }^{2}$ In contrast, especially increased contralesional and extramotor network activations have been correlated with decreased motor abilities. ${ }^{3,4}$ Previously termed lacunar infarcts (now preferably called recent small subcortical infarcts) ${ }^{5}$ represent a morphologically well-characterized subtype of stroke that may cause such impairments in mobility and cognition. ${ }^{1,6,7}$ More than $60 \%$ of patients with lacunar stroke still have motor dysfunction after 12 months. ${ }^{8}$

In contrast to other studies, we thus examined training effects on mobility and cognition in healthy controls (HCs) and patients with lacunar stroke, because this patient group has a higher chance for intact compensatory networks and residual impairments
Correspondence: Daniela Pinter Research Unit for Neuronal Repair and Plasticity, Department of Neurology, Medical University of Graz, Auenbruggerplatz 22, $8036 \mathrm{Graz}$, Austria $\mathrm{Tel}+4331638516437$

Email daniela.pinter@medunigraz.at 
of mobility. ${ }^{9}$ We here explored 1) the behavioral effects of a dedicated mobility training on i) motor and ii) cognitive abilities in chronic stroke patients with residual gait impairment and 2) whether parallel changes in cerebral activation measured by functional magnetic resonance imaging (fMRI), using an apparatus for bipedal ankle movements, occur.

\section{Patients and methods}

\section{Patients}

We included eight patients from our stroke outpatient department, aged between 69 and 81 years, who were willing to undergo a 5-week training and who satisfied the following inclusion criteria: mobility impairment (impaired gait function - three with a right-sided paresis and five with a left-sided paresis) caused by an imaging-proven ischemic subcortical infarct, which had occurred at least 6 months (mean time interval $=3.7$ years, range $=2-4$ years) before inclusion. Mobility impairment was defined by $\mathrm{m} / \mathrm{s}$ in the 10 meter walk test ([10 MWT] scores $<0.93 \pm 0.64 \mathrm{~m} / \mathrm{s}) .{ }^{10}$ One patient used a cane for longer distances, but all reported difficulties in walking long distances, such as the need for pauses and decreased strength and security. Three patients had a left-hemispheric lacunar subcortical infarct (located in the corona radiata or basal ganglia), four patients had a right-hemispheric lacunar subcortical infarct (located in the capsula interna, thalamus, or basal ganglia), and a single patient had a right-sided pontine lacunar infarct. Patients were not considered for participation if there was a contraindication for MRI or mobility training (eg, orthopedic or rheumatic diseases) or if there were severe cognitive (Montreal Cognitive Assessment [MoCa] score of <27), affective (Geriatric Depression Scale [GDS] score of $>5$ ) or sensory impairments, which could have affected engagement in the study or other competing medical disorders.

\section{Controls}

As controls (HCs), we included 16 healthy people in the same age range (between 62 and 80 years). Exclusion criteria were analogous to those in patients.

The Ethics committee of the Medical University of Graz approved the study and all participants gave informed written consent.

\section{Intervention}

During the 5 consecutive weeks of training, patients received 15 training units, thrice per week, with each domain lasting for 20-30 minutes (including balance, strength, and endurance), rendering a total training time per participant of 1,350 minutes (22.5 hours). The training was guided by experienced physical therapists at a geriatric institution, the Albert Schweitzer Clinic, Graz, and supervised by physicians experienced in neurorehabilitation and geriatric medicine. The study design is shown in Figure 1.

\section{Behavioral outcome assessment}

At baseline and follow-up after 5 weeks, study participants underwent clinical examination, assessment using two scales $\left(\mathrm{GDS}^{11}\right.$ and WHO Quality of Life [WHOQOL] ${ }^{12}$ ), and were neuropsychologically assessed using the following six tests: the Wechsler Memory Scale-logical memory (WMS-LM) and the delayed recall (WMS-LM DR), ${ }^{13}$ the Symbol Digit Modalities Test (SDMT), ${ }^{14}$ the MoCa,${ }^{15}$ the Trail Making Test A (TMT-A), and the Trail Making Test B (TMT-B). ${ }^{16}$

The GDS consists of 15 items to assess depressive mood, with higher scores indicating a higher degree of depression. The WHOQOL (0-100) measures four subtypes of quality of life, with higher scores indicating a higher quality. The WMS-LM consists of two stories that have to be reproduced instantly and at the end (WMS-LM DR) of neuropsychological assessment to measure logical memory. More reproduced items indicate better logical memory. The SDMT measures processing speed, with higher scores of solved items indicating higher processing speed. The MoCa consists of eight domains to test global cognition, with higher scores indicating higher cognitive abilities. The TMT-A measures attention, with lower processing time indicating a higher degree of attention. The TMT-B measures executive functions, with lower processing time indicating better executive functions.

Participants also underwent a 3-minute walking test and mobility assessment using the Demmi (De Morton Mobility Index $)^{17}$ and the Tinetti ${ }^{18}$ scales. Higher scores in the mobility scales indicate better mobility.

\section{Magnetic resonance imaging}

Structural and functional MRI data were acquired on a 3 Tesla Tim Trio Scanner (Siemens, Erlangen, Germany) using a 32-channel head coil. A high-resolution T1-weighted structural image (TR $=1,900 \mathrm{~ms}$, TE $=2.63 \mathrm{~ms}, 1 \mathrm{~mm}$ isotropic resolution, 176 slices) was acquired at baseline to allow for functional image registration, precise localization of activation, and location of old infarcts. Furthermore, a T2-weigthed fluid-attenuated inversion recovery (FLAIR) sequence $(\mathrm{TR} / \mathrm{TE} / \mathrm{TI}=10,000 / 69 / 2,500 \mathrm{~ms}$, in plane resolution $=0.9 \times 0.9 \mathrm{~mm}^{2}$, slice thickness $=3 \mathrm{~mm}$, no gap, slices $=44$ ) served to assess deep white matter hyperintensities 


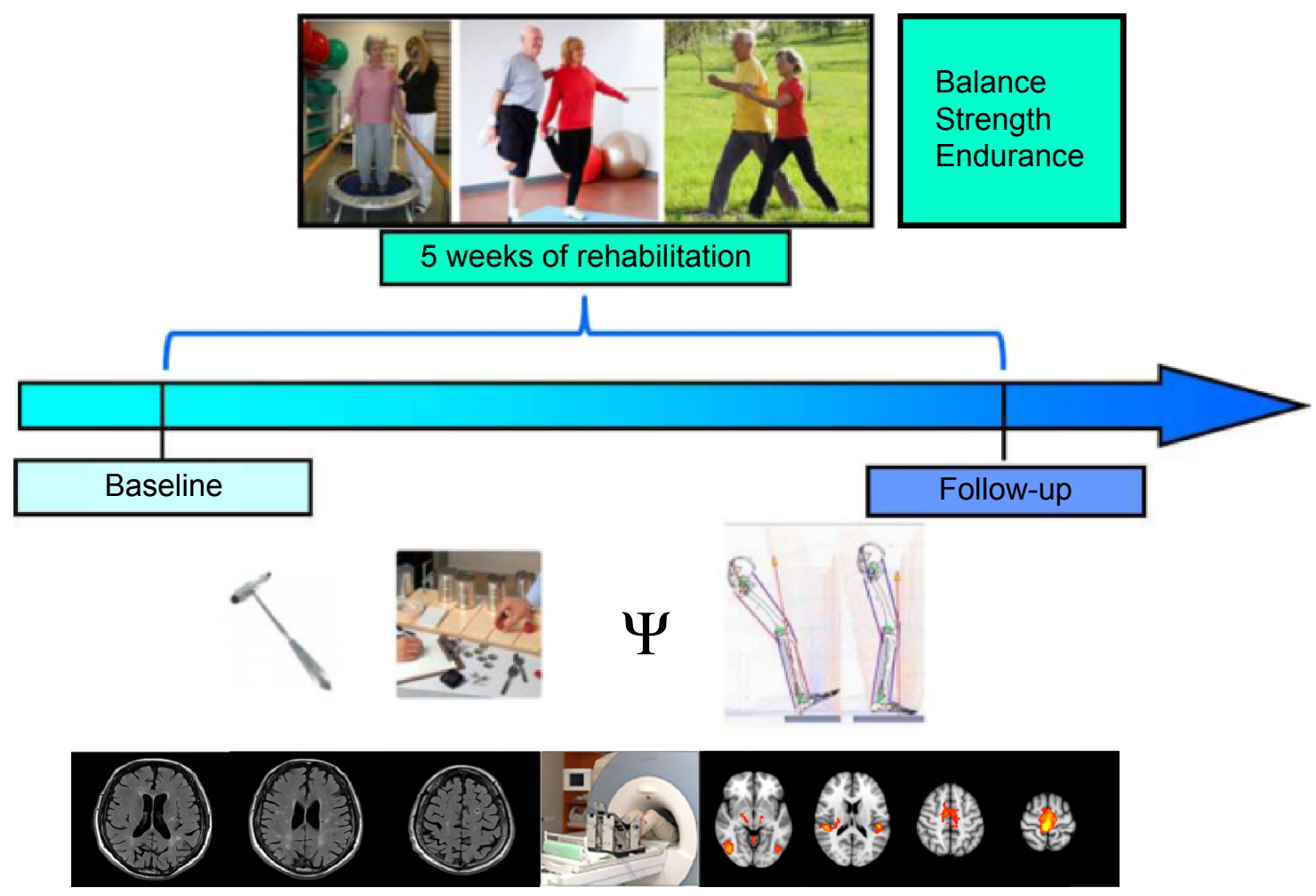

Figure I Design of the intervention study in chronic stroke patients undergoing 5 weeks of ambulatory training focusing on gait and coordination. Note: At baseline and follow-up, all participants underwent clinical, neuropsychological, and gait assessments and brain MRI.

Abbreviation: $\mathrm{MRI}$, magnetic resonance imaging.

$(\mathrm{WMH})$, which were classified using the Fazekas scale ${ }^{19}$ (grade $0=$ no lesions, $1=$ punctuate lesions, $2=$ early confluent lesions, and $3=$ confluent lesions). fMRI data were acquired on two occasions, before and after training, using identical scanning parameters $(\mathrm{TR}=3,000 \mathrm{~ms}, \mathrm{TE}=30 \mathrm{~ms}$, slices $=143$ ) and the same paradigm with the same scanner.

\section{fMRI paradigm}

A "block design", comprising bipedal ankle movements and periods of rest, modified based on paradigms used successfully in earlier studies, ${ }^{4,20,21}$ was employed. Bipedal movements were paced by arrows presented on a screen. Patients moved their feet alternately to simulate gait closely as possible. Vision was corrected with prism lenses if necessary. Blocks of bipedal ankle movement of 30 seconds alternated with periods of rest (21 seconds). Each session included four bipedal movement blocks separated by four blocks of rest. The paradigm was programmed in Presentation 16.3. The total scanning time for functional and structural imaging was 25 minutes. Prior to entering the scanner, subjects practiced the paradigm. Visual cues for ankle movements were presented on a screen and the experimenter explained the condition, conducting a training including the same purpose-built apparatus outside the scanner.

\section{fMRI data analysis}

Functional imaging analysis was carried out using FEAT (fMRI Expert Analysis Tool; Version 6.00, part of FMRIB's Software Library, www.fmrib.ox.ac.uk/fsl). The following prestatistics were applied: high pass filtering using a cutoff of 100 seconds; motion correction using MCFLIRT; brain extraction was done using Brain Extraction Tool; and spatial smoothing was performed using a Gaussian kernel of $5 \mathrm{~mm}$ full width at half maximum. Time-series statistical analysis was carried out using FMRIB's Improved Linear Model. Registration to high-resolution and/or standard images was carried out using FMRIB's Linear Image Registration Tool. Functional imaging data of the three patients with lefthemispheric strokes were flipped left to right by the midline, so all patients virtually had a right-sided stroke, as suggested previously.2. In first-level analysis, the effects of the bipedal movement blocks were determined for each subject. Head motion was corrected for using the displacement of the functional images in any direction, as derived from the FEAT motion correction report. Higher-level analysis was done using FLAME 1 (FMRIB's Local Analysis of Mixed Effects). $Z$-statistic images were thresholded using clusters determined by $Z>2.0$ for mean activations of bipedal movement and 
for post- versus precomparisons, with a corrected cluster significance threshold of $P<0.05 .{ }^{23}$ To examine functional changes between and within groups, we calculated unpaired and paired $t$-tests in second-level analysis.

\section{General statistical analysis}

Comparisons between patients and controls regarding behavioral and demographic data (mobility, neuropsychological assessment) were computed with the Statistical Package of Social Science (IBM SPSS Statistics 20; IBM Corporation, Armonk, NY, USA). The level of significance was set at 0.05. Baseline differences between groups and pre-post comparisons for each group were calculated with analysis of variance.

\section{Results}

\section{Clinical features of the cohort}

All participants were right handed. Patients and controls did not differ significantly with regard to sex, but controls tended to be younger $\left(\chi_{\operatorname{sex} 1,17}^{2}=0.486, P=0.667 ; \mathrm{F}_{\text {age } 1,22}=4.097\right.$, $P=0.055)$. HCs had a higher level of education $\left(F_{1,22}=4.403\right.$, $\left.P<0.05, \eta^{2}=0.17\right)$. Patients had mildly to moderately impaired walking abilities (10-meter walk: $\mathrm{MW}=0.9 \mathrm{~m} / \mathrm{s}$, $\mathrm{SD}=0.21 \mathrm{~m} / \mathrm{s})$. Patients tended to have a higher lesion WMH grade $\left(F_{1,22}=4.15, P=0.054\right)$ but lower executive functions $\left(F_{1,22}=4.45, P<0.05, \eta^{2}=0.17\right)$ and processing speed $\left(F_{1,22}=15.39, P<0.05, \eta^{2}=0.41\right)$ at baseline (details listed in Table 1 and shown in Figure 2).

\section{Behavioral outcome assessment after training}

After the intervention, patients showed increased mobility (Demmi $F_{1,7}=9.50, P<0.05, \eta^{2}=0.58$, Tinetti $F_{1,7}=4.59$, $P=0.07)$. They also showed improved logical memory $\left(F_{1,7}=5.67, P<0.05, \eta^{2}=0.48\right)$ and logical memory recall $\left(F_{1,7}=21.06, P<0.05, \eta^{2}=0.75\right)$. Table 2 lists the remaining nonsignificant results.

\section{Changes in cerebral activation in patients after training}

Subsequent to training, patients revealed increased activation in the right precentral gyrus, the right and left superior frontal gyri, and the right frontal lobe, with bipedal ankle movements (Figure 2). Controls showed no significant changes in activation with the paradigm. At baseline, patients (compared to controls) showed higher activation in the contralesional hemisphere (postcentral gyrus, parietal operculum, superior temporal gyrus, and left cerebellum) and decreased activation in the ipsilesional precentral gyrus and supplemental motor area. After training, these differences disappeared.

\section{Discussion}

In this exploratory study, we found potential changes in mobility, cognition, and cerebral activation after 5 weeks of ambulatory training focusing on gait and coordination in chronic stroke patients with residual gait disturbance.

Table I Baseline comparisons between chronic stroke patients and HCs

\begin{tabular}{|c|c|c|c|c|}
\hline & \multirow{2}{*}{$\frac{\text { Patients }(n=8)}{M(S D)}$} & \multirow{2}{*}{$\frac{\text { Controls }(n=16)}{M(S D)}$} & \multirow[t]{2}{*}{$P$-value } & \multirow[t]{2}{*}{$\eta^{2}$} \\
\hline & & & & \\
\hline \multicolumn{5}{|l|}{ Demographics } \\
\hline Sex, female, n (\%) & $5(63 \%)$ & $7(44 \%)$ & 0.67 & 0.17 \\
\hline Age (years) & $73.2(4.3)$ & $68.8(5.3)$ & 0.06 & 0.16 \\
\hline Education (years) & $12.7(4.1)$ & $16.0(3.3)$ & $0.048^{* *}$ & 0.17 \\
\hline WMH grade (Fazekas scale) 2/3, n (\%) & $3(37.5 \%)$ & $0(0 \%)$ & 0.055 & 0.16 \\
\hline \multicolumn{5}{|l|}{ Mobility } \\
\hline Mobility (Demmi) & 75.38 (12.09) & $100(0.00)$ & $0.00 * *$ & 0.76 \\
\hline Mobility (Tinetti) & $23.88(3.87)$ & $28.00(0.00)$ & $0.00 * *$ & 0.46 \\
\hline Speed (m/3 min) & $193.25(49.77)$ & $233.70(29.73)$ & $0.02^{* *}$ & 0.22 \\
\hline \multicolumn{5}{|l|}{ Cognition } \\
\hline Memory (WMS-LM) & $24.88(4.50)$ & $25.94(9.75)$ & 0.78 & 0.00 \\
\hline Processing speed (SDMT) & $29.12(6.94)$ & $44.75(10.08)$ & $0.00 * *$ & 0.41 \\
\hline Overall cognition (MoCa) & $26.50(1.69)$ & $27.56(1.79)$ & 0.18 & 0.08 \\
\hline Attention (TMT-A) & $47.63(15.91)$ & $38.88(14.74)$ & 0.20 & 0.07 \\
\hline Executive function (TMT-B/A) & $2.82(1.01)$ & $2.04(0.78)$ & $0.046 * *$ & 0.17 \\
\hline Memory (WMS-LM DR) & $19.75(4.56)$ & $21.50(8.16)$ & 0.63 & 0.01 \\
\hline Depression (GDS) & $2.25(1.39)$ & $0.31(0.60)$ & $0.00 * *$ & 0.51 \\
\hline Quality of life (WHOQOL) & $69.32(12.74)$ & $85.39(10.19)$ & $0.00 * *$ & 0.34 \\
\hline
\end{tabular}

Note: $* * P<0.05$.

Abbreviations: HCs, healthy controls; M, mean; SD, standard deviation; $\eta^{2}$, effect size; WMH, white matter hyperintensities; WMS-LM, Wechsler Memory Scale-Logical Memory; SDMT, Symbol Digital Modalities Test; MoCa, Montreal Cognitive Assessment; TMT, Trail Making Test A and B; WMS-LM DR, Wechsler Memory Scale-Logical Memory delayed recall; GDS, Geriatric Depression Scale; WHOQOL, World Health Organization's Quality of life questionnaire. 


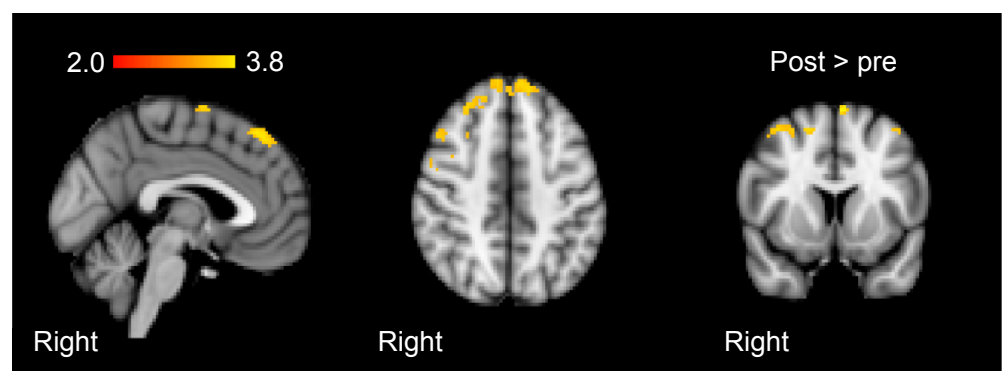

Figure 2 Group-mean activation changes from baseline to follow-up after training in the patient group.

Notes: Activations elicited by bipedal ankle movements. Images are presented in sagittal, axial, and coronal orientation in radiological convention. Clusterpeak was at $Z_{\max }=2.88, X=24, Y=26, Z=54$.

We noted mobility increases after training in our sample of chronic subcortical stroke patients, in line with an earlier study indicating that balance and basic motor abilities may be enhanced even in such "chronic" states. ${ }^{22}$ Conforming previous reports, cognition can be augmented not only through cognitive but also through mobility training and that, in turn, cognitive resources are needed for adequate motor activity and mobility. ${ }^{10,24,25}$ Supporting this notion, patients demonstrated improved memory after the training. Although causality with the training cannot be deferred and

Table 2 Changes from baseline to follow-up after 5 weeks of training in the patients

\begin{tabular}{|c|c|c|c|c|}
\hline & \multirow{2}{*}{$\frac{\text { Baseline }}{M \text { (SD) }}$} & \multirow{2}{*}{$\frac{\text { Follow-up }}{M(S D)}$} & \multirow[t]{2}{*}{$P$-value } & \multirow[t]{2}{*}{$\eta^{2}$} \\
\hline & & & & \\
\hline \multicolumn{5}{|l|}{ Mobility } \\
\hline \multicolumn{5}{|c|}{ Mobility (Demmi) } \\
\hline Patients & 75.38 (12.09) & $80.50(10.35)$ & $0.02^{* *}$ & 0.58 \\
\hline Controls & $100(0.00)$ & $100(0.00)$ & & \\
\hline \multicolumn{5}{|c|}{ Mobility (Tinetti) } \\
\hline Patients & $23.88(3.87)$ & $25.75(1.91)$ & $0.07 *$ & 0.40 \\
\hline Controls & $28.00(0.00)$ & $28.00(0.00)$ & & \\
\hline \multicolumn{5}{|c|}{ Speed $(\mathrm{m} / 3 \mathrm{~min})$} \\
\hline Patients & I 93.25 (49.77) & $192.00(33.65)$ & 0.92 & 0.00 \\
\hline Controls & $233.70(29.73)$ & $230.56(27.89)$ & 0.51 & 0.03 \\
\hline \multicolumn{5}{|c|}{ Cognition } \\
\hline \multicolumn{5}{|c|}{ Memory (WMS-LM) } \\
\hline Patients & $24.88(5.00)$ & $29.75(6.21)$ & $0.049 * *$ & 0.45 \\
\hline Controls & $25.94(9.75)$ & $30.69(8.50)$ & $0.01 * *$ & 0.38 \\
\hline \multicolumn{5}{|c|}{ Processing speed (SDMT) } \\
\hline Patients & $29.12(6.94)$ & $31.50(6.78)$ & 0.14 & 0.28 \\
\hline Controls & $44.75(10.08)$ & $43.44(8.00)$ & 0.34 & 0.06 \\
\hline \multicolumn{5}{|c|}{ Overall cognition (MoCa) } \\
\hline Patients & $26.50(1.69)$ & $27.88(1.64)$ & 0.15 & 0.28 \\
\hline Controls & $27.56(1.79)$ & $28.00(1.59)$ & 0.42 & 0.04 \\
\hline \multicolumn{5}{|c|}{ Attention (TMT-A) } \\
\hline Patients & $47.63(15.91)$ & 41.63 (II.17) & 0.28 & 0.17 \\
\hline Controls & $38.88(14.74)$ & $29.38(6.82)$ & $0.01 * *$ & 0.40 \\
\hline \multicolumn{5}{|c|}{ EF (TMT-B/A) } \\
\hline Patients & $2.82(1.01)$ & $2.84(1.38)$ & 0.98 & 0.00 \\
\hline Controls & $2.04(0.78)$ & $2.24(0.70)$ & 0.16 & 0.13 \\
\hline \multicolumn{5}{|c|}{ Memory (WMS-LM DR) } \\
\hline Patients & $19.75(4.56)$ & $27.63(4.8 I)$ & $0.00 * *$ & 0.75 \\
\hline Controls & $21.50(9.42)$ & $28.50(9.11)$ & $0.00 * *$ & 0.55 \\
\hline \multicolumn{5}{|c|}{ Depression (GDS) } \\
\hline Patients & 2.25 (I.39) & $2.75(1.58)$ & 0.17 & 0.25 \\
\hline Controls & $0.31(0.60)$ & $0.38(0.72)$ & 0.72 & 0.01 \\
\hline \multicolumn{5}{|c|}{ Quality of life (WHOQOL) } \\
\hline Patients & $69.32(12.74)$ & $66.54(16.90)$ & 0.48 & 0.07 \\
\hline Controls & $85.39(10.19)$ & $86.19(10.61)$ & 0.53 & 0.03 \\
\hline
\end{tabular}

Notes: $* * P<0.05, * P<0.10$. Respective data from $\mathrm{HCs}$ are given to indicate variability of the measures.

Abbreviations: M, mean; SD, standard deviation; HC, healthy control; $\eta^{2}$, effect size; WMS-LM, Wechsler Memory Scale-Logical Memory; SDMT, Symbol Digital Modalities Test; MoCa, Montreal Cognitive Assessment; TMT, Trail Making Test A and B; WMS-LM DR, Wechsler Memory Scale-Logical Memory delayed recall; GDS, Geriatric Depression Scale; WHOQOL, World Health Organization's Quality of life questionnaire; EF, executive function. 
test-retest effects need to be considered, this observation deserves closer consideration in future studies. Moreover, we did not specifically select patients with impaired cognition in our study, although spontaneous improvements in cognitive abilities in the chronic stage of stroke appear unlikely. ${ }^{25-27}$ Indeed, in line with the reported profile of cognitive changes after symptomatic subcortical infarcts, our patients also fared worse than controls regarding executive functions and processing speed at baseline.

In this study, we could not safely conclude on the effects of WMH on rehabilitation success because of the small sample size, which should be taken into consideration in future studies. It appears to be of interest in this context that a recent review on small vessel disease highlighted the possibility that the functional effects of WMH, eg, on cognition, might be obscured due to an increased cognitive reserve measured by education years in some individuals. ${ }^{28}$ This warrants further investigation with regard to training interventions.

On fMRI with bilateral ankle movements, patients demonstrated increased activation of the ipsi- and contralesional superior frontal gyri, ie, areas outside the motor network after the training. These brain regions have been implicated not only in self-consciousness, working memory, and executive functions but also in more complex motor functions..$^{29,30}$ Increases were also noted in the ipsilesional frontal lobe and ipsilesional precentral gyrus, which are considered as important areas for initializing and preparing motor actions. ${ }^{31}$ These results are partly in line with previous training studies, ${ }^{32-34}$ but mostly appear to indicate that the changes of brain function subsequent to a multimodal training focusing on such a complex behavior like gait but also affecting cognitive function may not be grasped by a simple motor paradigm. In this regard, using resting-state fMRI might be a superior approach as it obviates the need for restraining to a particular task and also leaves the possibility to assess changes in other networks. ${ }^{35,36}$ However, the fact that HCs who did not undergo training showed absolutely reproducible and unchanged cerebral activation at follow-up speaks for robustness of the used ankle movement fMRI paradigm.

Our study also has limitations. Importantly, the cognitive test battery should include validated parallel versions to avoid the learning effects that were also noted in our control group. The parallel versions should also be sensitive to educational differences between groups to measure only stroke or WMH-depending influences. These aspects need to be considered regarding the differences at baseline in cognition.

Furthermore, instead of testing a healthy cohort where ceiling effects are noted in the motor and gait tests, inclusion of a high-risk control group enriched for vascular risk factors might be more representative of the population in target. Extending the methodological limitations regarding fMRI (as already discussed), given the behavioral cognitive effects seen, additional inclusion of a cognitive paradigm appears favorable to more closely assess the effects of mobility training on cognitive brain function. In addition, more complex assessments like a dual task with walking while talking or providing cognitive challenges during gait assessment would be desirable to reveal more subtle deficits and effects.

It might also be possible that the overall results might have been biased, as our assessors were not blinded. However, patients were trained at the Albert Schweitzer Clinic, Graz, by experienced physiotherapists, whereas the MRI and behavioral outcome assessment took place at the Medical University of Graz by an unbiased researcher. In addition, social interaction might have increased motivation in our patients. Nevertheless, both groups were asked to daily record their physical activity for 5 weeks in a specific form. Thus, both groups focused on their physical function.

Because of our restricted inclusion criteria, we could only include a small sample size. Studying a larger cohort including randomization to intervention also comprising longer-term follow-up is mandatory to corroborate and extend our findings, with regard to the impact of preexisting brain damage as well. This should finally result in more refined insights regarding the effects of such a training and to predict responders. ${ }^{37}$

\section{Acknowledgments}

Part of this study was funded by a project of the country of Styria (project ID 4321) called "Untersuchungen zu den Effekten eines Trainings von Gang und Gleichgewicht auf Gehirnfunktion im Alter - Einblicke anhand der funktionellen Magnetresonanztomographie (fMRT)". The authors thank Karin Brodtrager for technical support and all involved persons from the Albert Schweitzer Clinic Graz, especially Antonella de Campo, MD; Martin Petio; and Katrin Glänzer. Deepest gratitude goes to all participants of this study.

\section{Disclosure}

The authors report no conflicts of interest in this work.

\section{References}

1. Langhorne P, Bernhardt J, Kwakkel G. Stroke rehabilitation. Lancet. 2011;377(9778):1693-1702.

2. Erickson KI, Hohmann T. Die Effekte von Alter und Training auf die kognitive Gesundheit. Zeitschrift für Sportpsychologie. 2013;20(1): 25-32.

3. Calautti C, Baron J-C. Functional neuroimaging studies of motor recovery after stroke in adults: a review. Stroke. 2003;34(6):1553-1566. 
4. Linortner P, Fazekas F, Schmidt R, et al. White matter hyperintensities alter functional organization of the motor system. Neurobiol Aging. 2012;33(1):197.

5. Wardlaw JM, Smith EE, Biessels GJ, et al. Neuroimaging standards for research into small vessel disease and its contribution to ageing and neurodegeneration. Lancet Neurol. 2013;12(8):822-838.

6. Kim JS, Bae YH. Pure or predominant sensory stroke due to brain stem lesion. Stroke. 1997;28(9):1761-1764.

7. Liman TG, Heuschmann PU, Endres M, Flöel A, Schwab S, Kolominsky-Rabas PL. Changes in cognitive function over 3 years after first-ever stroke and predictors of cognitive impairment and long-term cognitive stability: the Erlangen Stroke Project. Dement Geriatr Cogn Disord. 2011;31(4):291-299.

8. Rosamond W, Flegal K, Furie K, et al. Heart disease and stroke statistics - 2008 update: a report from the American heart association statistics committee and stroke statistics subcommittee. Circulation. 2008;117(71):25-146.

9. De Laat KF, Van Norden AG, Gons RA, et al. Gait in elderly with cerebral small vessel disease. Stroke. 2010;41(8):1652-1658.

10. Ward NS, Brown MM, Thompson AJ, Frackowiak RS. The influence of time after stroke on brain activations during a motor task. Ann Neurol. 2004;55(6):829-834.

11. Yesavage JA, Sheikh JI. Geriatric Depression Scale (GDS) recent evidence and development of a shorter version. Clin Gerontol. 1986; 5(1-2):165-173.

12. Development of the World Health Organization WHOQOL-BREF quality of life assessment. The WHOQOL group. Psychol Med. 1998;28(3):551-558.

13. Härting C, Markowitsch H, Neufeld H, Calabrese P, Deisinger K, Kessler J. Wechsler Gedächtnistest - Revidierte Fassung. WMS-R. (Manual). Bern, Switzerland: Hans Huber; 2000.

14. Rao S. A Manual for the Brief Repeatable Battery of Neuropsychological Tests in Multiple Sclerosis. Milwaukee, WI: Medical College of Wisconsin; 1990.

15. Nasreddine ZS, Phillips NA, Bédirian V, et al. The Montreal Cognitive Assessment, MoCA: a brief screening tool for mild cognitive impairment. J Am Geriatr Soc. 2005;53(4):695-699.

16. Tombaugh TN. Trail Making Test A and B: normative data stratified by age and education. Arch Clin Neuropsychol. 2004;19(2):203-214.

17. De Morton N, Davidson M, Keating JL. The de Morton Mobility Index (DEMMI): an essential health index for an ageing world. Health Qual Life Outcomes. 2008;6:63.

18. Marks D. Assessment: Tinetti-Test Sturzrisiko erkennen. Physiopraxis. 2006;2:32-33.

19. Fazekas F, Chawluk JB, Alavi A, Hurtig H, Zimmerman R. MR signal abnormalities at 1.5T in Alzheimer's dementia and normal aging defciency. AJR Am J Roentgenol. 1987;149(2):351-356.

20. Enzinger C, Johansen-Berg H, Dawes H, et al. Functional MRI correlates of lower limb function in stroke victims with gait impairment Stroke. 2008;39(5):1507-1513.

21. Enzinger C, Dawes H, Johansen-Berg H, et al. Brain activity changes associated with treadmill training after stroke. Stroke. 2009;40(7): $2460-2467$.
22. Dawes H, Enzinger C, Johansen-Berg H, et al. Walking performance and its recovery in chronic stroke in relation to extent of lesion overlap with the descending motor tract. Exp brain Res. 2008;186(2):325-333.

23. Worsley KJ. Statistical analysis of activation images. Functional MRI: An Introduction to Methods. Oxford, UK; Oxford University Press; 2001:251-270

24. Raz N, Rodrigue KM. Differential aging of the brain: patterns, cognitive correlates and modifiers. Neurosci Biobehav Rev. 2006;30(6): 730-748.

25. Montero-Odasso M, Verghese J, Beauchet O, Hausdorff JM. Gait and cognition: a complementary approach to understanding brain function and the risk of falling. J Am Geriatr Soc. 2012;60(11):2127-2136.

26. Kluding PM, Tseng BY, Billinger SA. Exercise and executive function in individuals with chronic stroke: a pilot study. J Neurol Phys Ther. 2011; 35(1):11-17.

27. Quaney B, Boyd L. Aerobic exercise improves cognition and motor function poststroke. Neurorehabil Neural Repair. 2009;23(9):879-885.

28. Pinter D, Enzinger C, Fazekas F. Cerebral small vessel disease, cognitive reserve and cognitive dysfunction. J Neurol. 2015;262(11): 2411-2419.

29. Wen W, Zhu W, He Y, et al. Discrete neuroanatomical networks are associated with specific cognitive abilities in old age. JNeurosci. 2011; 31(4):1204-1212.

30. Martino J, Gabarrós A, Deus J, et al. Intrasurgical mapping of complex motor function in the superior frontal gyrus. Neuroscience. 2011; 179:131-142.

31. Porro CA, Francescato MP, Cettolo V, et al. Primary motor and sensory cortex activation during motor performance and motor imagery: a functional magnetic resonance imaging study. JNeurosci. 1996;16(23): 7688-7698.

32. Scholz J, Klein MC, Behrens TE, Johansen-Berg H. Training induces changes in white-matter architecture. Nat Neurosci. 2009; 12(11):1370-1371.

33. Bosnell RA, Kincses T, Stagg CJ, et al. Motor practice promotes increased activity in brain regions structurally disconnected after subcortical stroke. Neurorehabil Neural Repair. 2013;25(7):607-616.

34. Carey LM, Abbott DF, Egan GF, et al. Evolution of brain activation with good and poor motor recovery after stroke. Neurorehabil Neural Repair. 2006;20(1):24-41.

35. Ovadia-Caro S, Villringer K, Fiebach J, et al. Longitudinal effects of lesions on functional networks after stroke. J Cereb Blood Flow Metab. 2013;33(8):1279-1285.

36. Westlake KP, Nagarajan SS. Functional connectivity in relation to motor performance and recovery after stroke. Front Syst Neurosci. 2011 $5(8): 12$.

37. Nadkarni NK, Studenski SA, Perera S, et al. White matter hyperintensities, exercise, and improvement in gait speed: does type of gait rehabilitation matter? J Am Geriatr Soc. 2013;61(5):686-693.
Clinical Interventions in Aging

\section{Publish your work in this journal}

Clinical Interventions in Aging is an international, peer-reviewed journal focusing on evidence-based reports on the value or lack thereof of treatments intended to prevent or delay the onset of maladaptive correlates of aging in human beings. This journal is indexed on PubMed Central, MedLine,

\section{Dovepress}

CAS, Scopus and the Elsevier Bibliographic databases. The manuscript management system is completely online and includes a very quick and fair peer-review system, which is all easy to use. Visit http://www.dovepress. com/testimonials.php to read real quotes from published authors. 